Management options

\title{
The role of surgery in pseudomembranous enterocolitis
}

\author{
Y K S Viswanath, C D M Griffiths
}

\begin{abstract}
Summary
Pseudomembranous enterocolitis is an inflammatory bowel disorder caused by Clostridium difficile toxins. Classical presentation includes abdominal pain, pyrexia, diarrhoea and leucocytosis. The management is mainly conservative but in extreme cases surgery is necessary. Resectional procedures (colectomy) carry a better prognosis than diversion procedures (colostomy). A careful history, a high index of suspicion, and early diagnosis and treatment would reduce the associated morbidity and mortality of this condition. The aetiopathogenesis, pathology, clinical presentation, diagnosis, differential diagnoses, complications, medical and surgical management are reviewed, and three case reports briefly discussed. A management algorithm is also suggested.
\end{abstract}

Keywords: pseudomembranous enterocolitis; Clostridium difficile toxins; surgery

\section{PMEC: predisposing factors \\ - antibiotics \\ - malignant obstruction \\ - intestinal ischaemia \\ - renal failure \\ - debilitating illness \\ - Hirschsprung's disease in children \\ - systemic chemotherapy}

Box 1
Pseudomembranous enterocolitis (PMEC) was first described by Dr Finny in $1893,{ }^{12}$ but it was not until 1978 that Clostridium difficile and its toxins were isolated and identified as the cause. ${ }^{2} C$ difficile is a Gram-positive, spore-bearing anaerobic bacillus, which may be present as a part of normal bacterial flora in the gut. ${ }^{3-5}$ The disease spectrum of $C$ difficile infection may range from antibiotic-associated diarrhoea, and antibiotic-associated colitis, to its most severe form, PMEC. ${ }^{1367}$ The incidence of $C$ difficile infection has increased sixfold in England and Wales in the past five years. ${ }^{5}$

\section{Aetiopathogenesis}

$C$ difficile overgrowth due to alteration of bacterial gut flora and subsequent release of exotoxins induces the characteristic inflammatory changes of PMEC. ${ }^{1-3}{ }^{6}$ Prior antibiotic exposure is the single important risk factor in the causation of PMEC. All commonly used antibiotics, including ampicillin, amoxycillin, tetracycline and cephalosporins, have been implicated in its pathogenesis ${ }^{136-12}$ Other predisposing factors are mentioned in box $1 .^{1-368-10}$ The two important exotoxins released by $C$ difficile are toxin $A$ (enterotoxin) and toxin B (cytotoxin), which induce the inflammatory process by their enteropathogenic and cytopathic effects, respectively..$^{1-36} 6910$

\section{Pathology}

PMEC usually involves the colon in its entire length, although it can involve only a portion. ${ }^{210} 14$ Occasionally it may extend to the small bowel, hence the term enterocolitis. ${ }^{21014}$ The characteristic macroscopic features are the presence of multiple green to yellowish plaques on the mucosal surface (figure 1). The size of the plaque may vary from a few millimetres to a confluent patch..$^{127}$ Each plaque represents a pseudomembrane which, on microscopy, shows the presence of mucous inflammatory cells and fibrin exudate covering an area of necrosis (figure 2). This process of inflammation has been described as fibrinoid necrosis. ${ }^{127}$ The underlying crypts may become dilated and disrupted in late stages (figure 2B). ${ }^{5}$ Important complications ${ }^{168121516}$ and differential diagnoses $^{11516}$ of PMEC are depicted in boxes 2 and 3, respectively.

PMEC: complications
Metabolic
- severe dehydration with electrolyte imbalance
- hypoalbuminaemia
Surgical
- toxic megacolon
- colonic perforation and peritonitis
- paralyaemia and secondary endotoxic shock
- multisystem organ failure leading to death

Box 2

\section{Clinical features}

PMEC commonly presents with moderate to profuse diarrhoea, abdominal pain and tenderness, pyrexia, and tachycardia. ${ }^{1238}$ Passage of blood in the stools is infrequent, but may occur in fulminant cases. ${ }^{12}$ If the disease is
Correspondence to Mr YKS Viswanath, Flat 2 NMA, South Tyneside General Hospital, Harton Lane, Southshields NE34 OPL, UK

Accepted 23 September 1997 


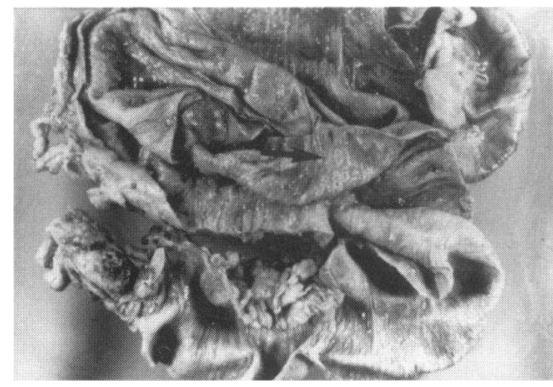

Figure 1 Cut section of a colectomy specimen with multiple discrete plaques (straight arrow) on the mucosal surface

\section{PMEC: differential diagnoses}

- acute ulcerative colitis

- Crohn's disease

- gastroenteritis, including Salmonella infections

- ischaemic colitis

- amoebic colitis

Box 3

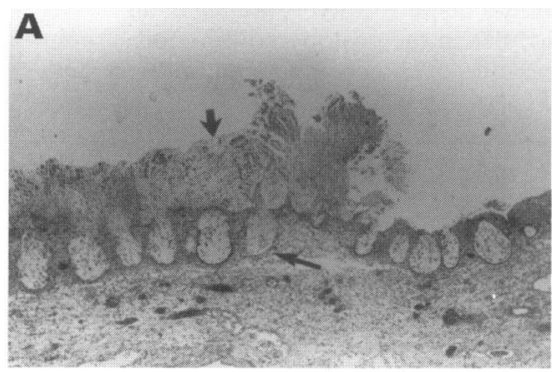

B

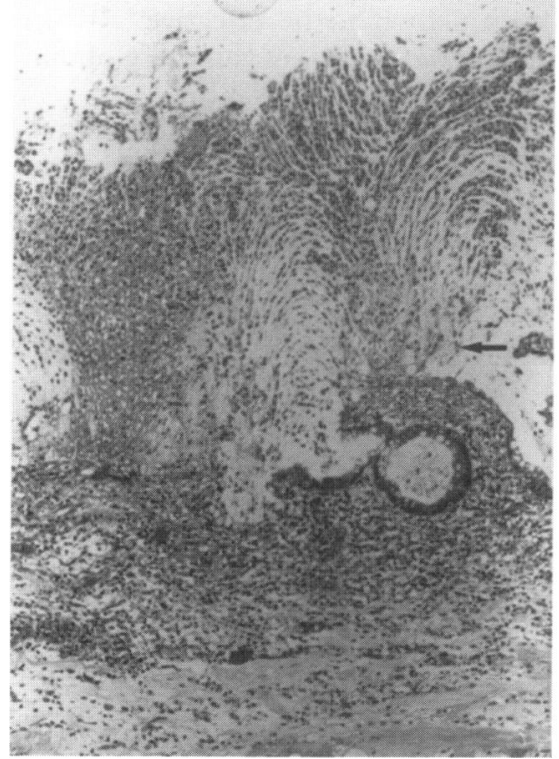

Figure 2 (A) Photomicrograph showing multiple distorted crypts (long straight arrow) with an overlying membrane (short straight arrow) of inflammatory debris (original magnification $\times 100$ ). (B) Close-up of a disrupted crypt with a fountain-like (straight arrow) fibrin exudate (original magnification $\times 250$ ) localised to the caecum and the ascending colon, it may present with features of acute appendicitis, leading to an unnecessary laparotomy. ${ }^{11}$ Boxes 4,5 and 6 contain summaries of three pertinent case reports.

\section{Case report 1}

A 72-year-old woman presented with acute onset of abdominal pain with features of peritonitis. She had had a seven-day course of amoxycillin to treat chest infection in the two weeks preceding admission. Investigations revealed a markedly raised white cell count of $69.8 \times 10^{9} / 1$ with severe dilatation of the colon on plain abdominal X-ray. At surgery the colon was dilated and oedematous throughout with multiple areas of impending perforation. She underwent a sub-total colectomy with an ileostomy. Histology revealed extensive pseudomembranous colitis with an incidental Dukes' A adenocarcinoma in the right colon. She made an uneventful recovery.

\section{Box 4}

\section{Case report 2}

A 66-year-old woman underwent an elective open cholecystectomy uneventfully and had 24-h peri-operative antibiotic cover in the form of iv cefuroxime. She developed abdominal pain with distension associated with diarrhoea on the fourth postoperative day. Stool samples were positive for $C$ difficile toxin. Proctosigmoidoscopy showed features consistent with PMEC. A trial of conservative treatment including parenteral metronidazole failed and she became toxic with signs of peritonitis. Consecutive plain abdominal X-rays revealed progressive dilatation of the transverse and the descending colon. At emergency laparotomy, she was found to have a severe pancolitis and underwent a subtotal colectomy with an ileostomy. Postoperatively she developed signs of systemic sepsis and required intensive therapy unit admission for 10 days. She made a slow recovery and was subsequently discharged.

\section{Box 5}

\section{Case report 3}

A 67-year-old woman presented with a three-day history of increasing abdominal pain and fever. Abdominal examination showed signs of peritonitis with marked tenderness in the left lower quadrant. At laparotomy, the sigmoid colon was dilated and dusky with multiple black spots on the serosal surface. She underwent sigmoid colectomy with an end colostomy. Histology showed features of ischaemic colitis along with typical pseudomembranous plaques on the mucosal surface. The patient had an uneventful postoperative period and remained well in the follow-up period of six months.

\section{Box 6}

\section{Diagnosis}

The mainstay of diagnosis is the isolation of $C$ difficile and detection of exotoxins in the stool sample..$^{1-3} 79$ Stool examinations have been shown to be culture positive for $C$ difficile in $95 \%$ and toxin-positive in $97-100 \%$ of cases. ${ }^{136}$ The detection of toxin B (cytotoxin) in stools has been regarded as the single standard technique in the diagnosis of this condition ${ }^{1-3} 679$ Proctosigmoidoscopy will show typical pseudomembranes and may be normal in patients with localised disease. $^{1-3}$ Blood investigations usually reveal marked leucocytosis with neutrophilia. ${ }^{2}$ Plain X-ray of the abdomen may show colonic dilatation, with or without loss of haustrations (figure 3). ${ }^{15}{ }^{16} \mathrm{~A}$ barium enema should be avoided in emergency cases as it can precipitate toxic megacolon. ${ }^{16}$ Computed tomography may show colonic dilation and diffuse thickening of the colonic wall with a doughnut-like appearance. These changes are not specific for PMEC, but can play an important role in recognising this condition. ${ }^{13}$ 17-19

\section{Management}

The initial management of PMEC primarily consists of isolation of the patient, cessation of the precipitating antibiotics, and correction of fluid and electrolyte imbalance. ${ }^{136}$ Nearly $15 \%$ of patients respond to this approach. ${ }^{6}$ Specific therapy is indicated in the remaining $85 \%$ of cases. Vancomycin (oral $125 \mathrm{mg} 6$ 
Figure 3 (A) Plain abdominal X-ray showing the dilated transverse colon. (B) Plain abdominal $\mathrm{X}$-ray in a case of severe PMEC showing dilated small and large bowel
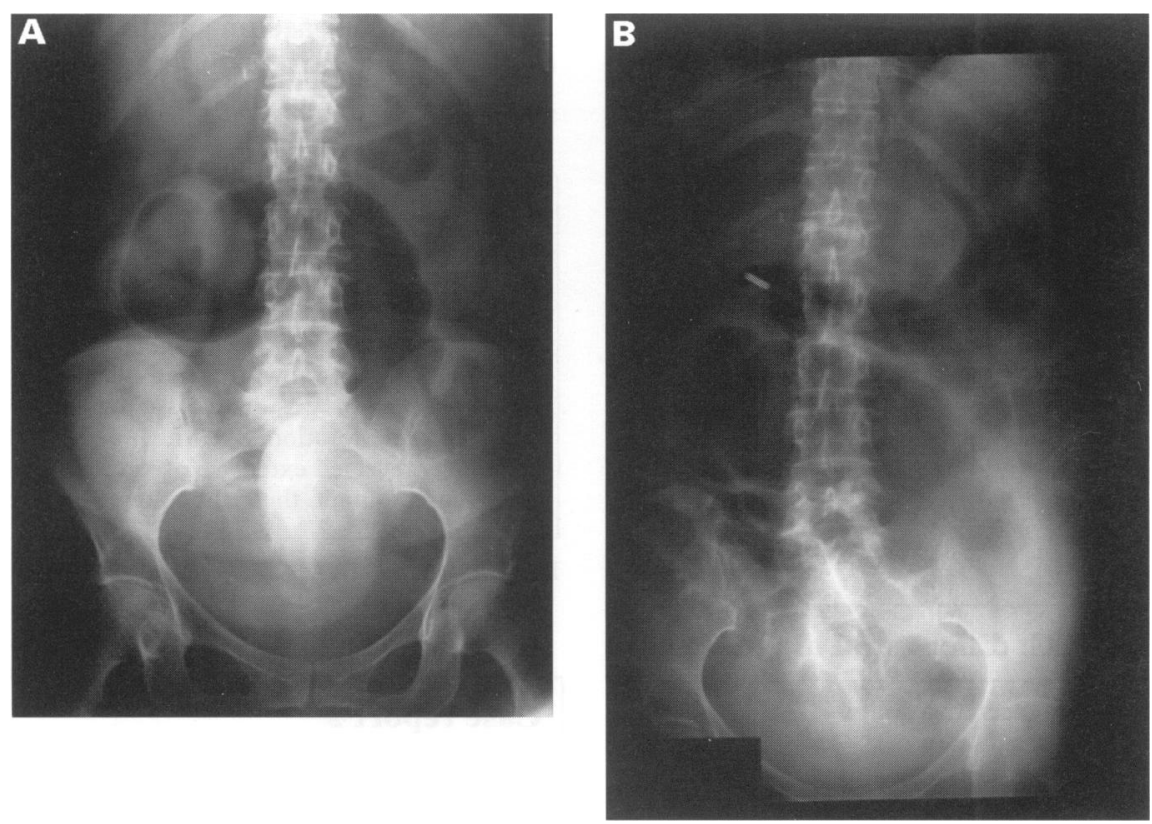

\section{Learning points}

- a careful history with a high index of suspicion, marked leucocytosis, and dilatation of the colon on plain abdominal film will give important clues to the diagnosis of PMEC and cohort nursing

b) Stop antibiotics

c) Correction of fluid and electrolyte imbalance

If systemically unwell,

(a) + (b)

$+(\mathrm{c})$ and

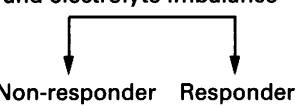

- PMEC can present as an acute abdomen

- stool examination and proctosigmoidoscopy should be performed routinely in all patients who develop abdominal pain with diarrhoea in the postoperative period

- subtotal colectomy with an ileostomy is the operation of choice in patients with pancolitis

Box 7

d) Specific therapy

(vancomycin or metronidazole)

No improvement after 7 days and/or clinical condition

is worsening

e) Surgical intervention

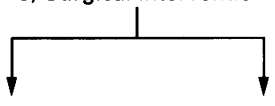

Resectional procedures (preferred and should be the first choice)

Diversion procedures (if the patient is poorly and not fit for a major resection)

- colostomy

- sub-total colectomy

- caecostomy

- ileostomy

Figure 4 Algorithm of the management of PMEC
1 Morris JB, Zollinger RM, Stellato TA. Role of surgery in antibiotic induced pseudomembranous enterocolitis. Am $\mathcal{f}$ Surg 1990;160:535-9.

2 O'Connor TW. Pseudomembranous enterocolitis: a historical and clinical review. Dis Colon Rectum 1981;24:445-8.
Bradbury AW, Barret S. Surgical aspects of Clostridium difficile colitis. $\mathrm{Br} \mathcal{7}$ Surg 1997;84: 150-9.

4 Cartmill TD, Panigrahi H, Worsley MA McCann DC, Nice CN, Keith E. Management and control of a large outbreak of diarrhoea due to Clostridium difficile. 7 Hosp Infect 1994;27:1-15.

5 Price AB. Infections of the large intestine. In McGee JO, Issacson PG, Wright NA, eds Oxford Textbook of pathology. Oxford: Oxford University Press, 1992; pp 1222-7. 
6 Wilcox MH. Clostridium difficile infection. Surg Infect Curr f Rev 1995;7:74-8.

7 Poxton IR, Brown R, Fraser AG, Collee JG. Enteropathogenic Clostridia and Clostridium botulinum. In: Collee JG, Marmion BP, Frase AG, eds. Mackie and McCartney's Practical medical microbiology. London: Churchill Livingstone, 1996; pp 537-47.

8 Bradley SJ, Weaver DW, Maxwell NP, Bouwman DL. Surgical management of pseudomembranous colitis. Am Surg 1988;54:329-32.

9 Meyer-Kawohi R, Bockemuhl J. Clostridium difficile enterocolitis, pathogenesis, clinical course epidemiology and laboratory diagnosis. Immun Infekt 1986;14:63-7.

10 Azmy AAF, Raine PAM, Young DG. Pseudomembranous enterocolitis-potentially lethal disease. F Paediatric Surg 1996;25:453-6.

11 Drapkin MS, Worthington MS, Chang TW, Razvi SA. Clostridium difficile colitis mimicking acute peritonitis. Arch Surg 1985;120:1321-3.

12 Osler T, Lott D, Bordley J 4th, Lynch F Ellsworth C, Kozak A. Cefazolin induced pseudomembranous colitis resulting in perforation of sigmoid colon. Dis Colon Rectum 1986;29:140-3.
13 Kamthan AG, Bruckner HW, Hirschman SZ Agus SG. Clostridium difficile diarrhoea induced by cancer chemotherapy. Arch Intern Med 1992; by cancer chem

14 Unushihara N, Kohno S, Hasegawa S. Pseudomembranous Enterocolitis and haemorrhagic necrotising enterocolitis in Hirschprung's disease. Surg Today 1994;24:221-4.

15 Thomford NR. Toxic megacolon. Surg Ann 1980;12;341-50.

16 Trudel JL, Deschnes M, Mayrand S, Barkun AN. Toxic megacolon complicating pseudomembranous colitis. Dis Colon Rectum 1995;38: 1033-8.

17 Letourneau JG, Day DL, Steely JW, Goldber ME. CT appearance of antibiotic induced colitis. Gastrointest Radiol 1987;12;257-61.

18 Mukai JK, Janower ML. Diagnosis of pseudomembranous colitis by computed tomography: membranous colitis by computed tomography: a report of two

19 Haaga JR, Bick RJ, Zollinger RM Jr. CT-guided percutaneous catheter caecostomy. Gastrointes Radiol 1987;12:166-8.

20 Wilcox MH, Howe R. Diarrhoea caused by Clostridium difficile: response time for treatmen with metronidazole and vancomycin. $\mathcal{F}$ Antimicrob Chemother 1995;36:673-9.
21 Gorbach SL, Chang TW, Goldin B. Successful treatment of relapsing Clostridium difficile colitis with Lactobacillus GG. Lancet 1987;2:1519.

22 Biller JA, Katz AJ, Flores AF, Bulie TM Gorbach SL. Treatment of recurrent Clostridium difficile colitis with Lactobacillus GG. $\mathcal{F}$ Paedia Gastroenterol Nutr 1995;21:125-9.

23 Leung DY, Kelly CP, Boguniewidz M, Pothoulakis C, LaMont JT, Flores A. Treatment with intravenously administered gamma globulin of chronic relapsing colitis induced by Clostridium difficile toxin. F Paediatr 1991;118:633-7.

24 Boyd WC, DenBeston L. Subtotal colectomy for refractory pseudomembranous enterocolitis. fAMA 1976;235:181.

25 Saylor JL, Anderson CB, Tedesco FJ. Pseudomembranous colitis treated with completely diverting ileostomy. Arch Surg 1976;111:596-8.

\section{Medical Anniversary}

\section{FRIEDRICH WEGENER, 7 April 1907}

Friedrich Wegener (1907-1990) was born in Varel, Oldenburg, Germany. His father was a doctor and his mother a Swedish gymnastic director. With this athletic background and a fine physique, he became, in 1931, German hammer-throwing champion. He graduated MD (1932) in Kiel with a thesis entitled Testicular tumours. He became a pathologist in Kiel, then Breslau, and finally Professor in Lubeck. He described three patients with his syndrome to the German Pathological Society (1936) and during his lifetime saw 12 cases of Wegener's granulomatosis but never used that eponym. He had seven children and 17 grandchildren when he died on 9 July 1990.

Wegener's granulomatosis involves nasopharynx, lungs, eyes and kidneys. It was universally fatal until the advent of corticosteroids, cyclophosphamide, and antibiotics. Wegener lived to see two remarkable advances in his disorder. Firstly, the introduction of a most helpful diagnostic blood test; anti-neutrophil cytoplasmic autoantibodies are identified in serum of patients by immunofluorescence microscopy using alcohol-fixed normal human neutrophils. He also derived considerable joy from the second advance, namely treatment. When he first described the condition in 1936 it was associated with $100 \%$ mortality. In the succeeding 50 years, he witnessed the introduction of corticosteroids, cyclophosphamide, and antibiotics, all of which have combined to reduce the mortality to $5-10 \%$. He was delighted that earlier diagnosis by a non-invasive procedure led to much earlier treatment and thus a substantial improvement in prognosis. - DG fames 